\title{
Ligand Discrimination in Myoglobin from
}

\section{Linear-Scaling DFT+ $U$}

\author{
Daniel J. Cole, ${ }^{\dagger}$ David D. O’Regan ${ }^{\dagger}$ and Mike C. Payne ${ }^{*, \dagger}, \mathbb{A}$ \\ Theory of Condensed Matter Group, Cavendish Laboratory, University of Cambridge, J. J. \\ Thomson Avenue, Cambridge CB3 OHE, UK., Theory and Simulation of Materials, École \\ Polytechnique Fédérale de Lausanne, MXC 341, Station 12, CH-1015 Lausanne, Switzerland., \\ and \\ E-mail:mcp1@cam.ac.uk
}

\footnotetext{
${ }^{*}$ To whom correspondence should be addressed

'University of Cambridge

*École Polytechnique Fédérale de Lausanne

${ }^{\mathbb{I}}$ This document is the Accepted Manuscript version of a Published Work that appeared in final form in J. Phys. Chem. Lett., 2012, 3 (11), pp 1448-1452, copyright (C) American Chemical Society after peer review and technical editing by the publisher.

To access the final edited and published work see http://pubs.acs.org/doi/full/10.1021/jz3004188
} 


\begin{abstract}
Myoglobin modulates the binding of diatomic molecules to its heme group via hydrogenbonding and steric interactions with neighboring residues, and is an important benchmark for computational studies of biomolecules. We have performed calculations on the heme binding site and a significant proportion of the protein environment (more than 1000 atoms) using linear-scaling density functional theory and the $\mathrm{DFT}+U$ method to correct for self-interaction errors associated with localized $3 d$ states. We confirm both the hydrogen-bonding nature of the discrimination effect $(3.6 \mathrm{kcal} / \mathrm{mol})$ and assumptions that the relative strain energy stored in the protein is low (less than $1 \mathrm{kcal} / \mathrm{mol}$ ). Our calculations significantly widen the scope for tackling problems in drug design and enzymology, especially in cases where electron localization, allostery or long-ranged polarization influence ligand binding and reaction.
\end{abstract}

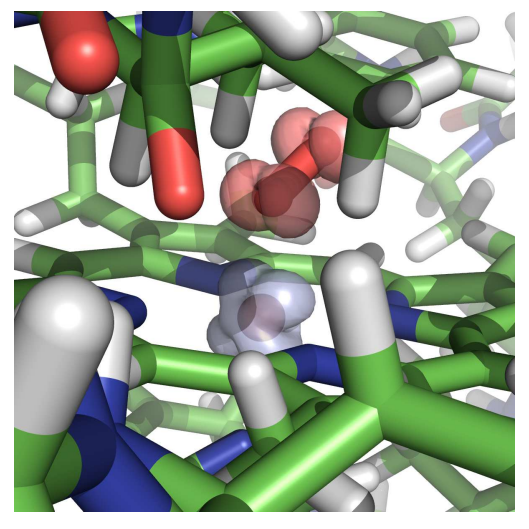

Table of contents graphic.

Keywords: Density functional theory, Hubbard U, myoglobin, heme, metalloprotein 
Myoglobin (Mb) is a small, globular protein, which is responsible for storing oxygen in muscle tissues. Mb contains a single heme group, which is packed within a predominantly $\alpha$-helical secondary structure and is co-ordinated by a histidine residue (known as the proximal histidine) as the fifth ligand of the heme's central iron ion. The $3 d$ electrons of the ferrous heme iron ion (Fe(II)) are energetically well-aligned with $\pi^{*}$ acceptor orbitals in $\mathrm{CO}$ and $\mathrm{O}_{2}$ and, as such, are capable of strongly binding these gaseous molecules. The Mb protein famously reduces the heme group's natural preference for $\mathrm{CO}$ binding: the binding energy of $\mathrm{CO}$, relative to $\mathrm{O}_{2}$, is reduced approximately 1000 -fold (or $\sim 4 \mathrm{kcal} / \mathrm{mol}$ ) in the protein environment. ${ }^{1}$ The influence of the protein is traditionally split into two effects, ${ }^{2}$ mediated by two distal protein residues H64 and V68 (1). First, the $\pi^{*}$ acceptor orbitals on $\mathrm{O}_{2}$ are lower in energy than on $\mathrm{CO}$, resulting in greater charge transfer from the Fe $3 d$ orbitals and, hence, a stronger electrostatic interaction with the neighboring H64. Second, the symmetry of unoccupied $\mathrm{CO} \pi^{*}$ acceptor orbitals results in a linear lowest energy binding conformation with heme: the $\mathrm{Fe}-\mathrm{C}-\mathrm{O}$ bond angle is close to $180^{\circ}$, while $\mathrm{Fe}-\mathrm{O}-\mathrm{O}$ is closer to $120^{\circ}$. It has been argued that steric interactions involving the H64 and V68 residues of myoglobin reduce the affinity of $\mathrm{CO}$ relative to $\mathrm{O}_{2}$, which can be more easily incorporated into the binding cavity in its lowest energy bent conformation..$^{3}$

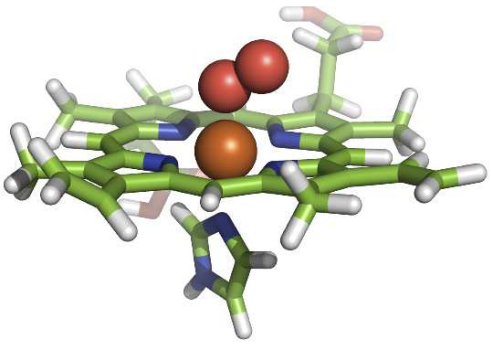

1 residue

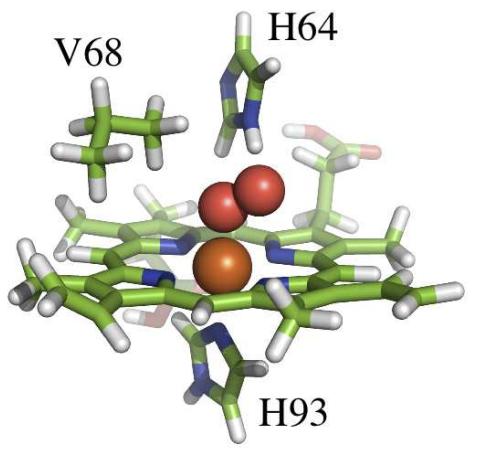

3 residues

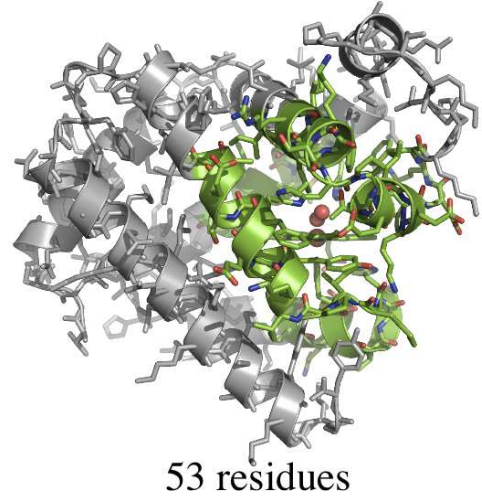

53 residues

Figure 1: Computational models used in the current study. Iron and oxygen ligand atoms are represented by orange and red spheres, respectively. Distal residues H64 and V68, identified as important in determining ligand binding, are labeled, as well as the proximal histidine H93. In the 53-residue system, the full myoglobin molecule is shown in grey, while the system used in our calculations is colored. 
The nature of the protein effect has been well-studied and makes myoglobin an important benchmark for biocatalysis studies and structure-function relationships. Density functional theory (DFT), ${ }^{4}$ QM/MM ${ }^{5}$ and Poisson-Boltzmann ${ }^{6}$ studies indicate that the first, hydrogen-bonding factor is the dominant one in determining preference for $\mathrm{O}_{2}$ binding in myoglobin. Site-directed mutagenesis of H64 to various hydrophobic residues implies that it contributes around $3.7 \mathrm{kcal} / \mathrm{mol}$ to ligand discrimination. ${ }^{\underline{7}}$ In conjunction with DFT studies showing that the strain energy stored in the $\mathrm{CO}$ ligand in more recent crystal structures is quite low $(<1 \mathrm{kcal} / \mathrm{mol}), \frac{2}{2}$ a consistent picture of ligand discrimination via hydrogen-bonding to the distal histidine emerges. However, as with many similar computational simulations of ligand binding and reactions in proteins, the protein environment of the heme group beyond a small number of residues is generally neglected or is included in a QM/MM description. This leaves two important questions unanswered. First, how does long-range polarization by the protein environment affect charge transfer to the $\mathrm{O}_{2}$ ligand and its electrostatic interactions with H64? Second, how much strain energy is stored in the protein itself, and does this factor affect its ability to discriminate sterically between ligands?

Here, we address these questions by means of linear-scaling DFT as implemented in the ONETEP ${ }^{8}$ code, which combines basis set accuracy equivalent to that of plane-wave DFT methods, with a computational cost that scales linearly with the number of atoms in the system. In combination with a DFT energy functional augmented by damped London terms capable of describing van der Waals interaction in weakly bound systems (DFT+D), $\underline{9}$ this method allows for an accurate, fully QM description of systems of thousands of atoms, $\underline{10}$ including entire proteins. $\stackrel{11,12}{\underline{H}}$ However, conventional local or semi-local approximate exchange-correlation functionals for DFT often fail to describe the physics of strongly localized orbitals, particularly those of $3 d$ symmetry, due to selfinteraction errors associated with the lack, or underestimation, of the exact functional's derivative discontinuity with respect to particle number. $\underline{13}$ Typical symptoms of self-interaction error may include, for example, underestimated localization and related local moments, spurious selection of low-spin states, underestimated single-particle and optical excitation energies, and qualitatively incorrect metal-ligand binding. The latter problem, in particular, inhibits the reliable application 
of such functionals to the study of the transition metal binding sites central to the function of a range of proteins. Since conventional, inexpensive functionals often perform acceptably well for describing lighter elements, and hybrid functionals comprising a fraction of exact exchange are excessively expensive for large systems, we favor the DFT + Hubbard $U(\mathrm{DFT}+U$, also known as $\mathrm{LDA}+U)$ approach for correcting the functional locally, thus canceling the self-interaction errors directly in the $3 d$ subspaces where they are most grave. The DFT $+U$ method is most well-known in solid-state physics, $\stackrel{14,15}{\underline{ }}$ but has been demonstrated to be particularly efficient and effective for correcting the self-interaction error in transition metal chemistry $\underline{16}-18$ and is used with increasing frequency for biological systems. The implementation of the DFT $+U$ method in the linear-scaling framework, with full optimization of both the local orbitals describing the Kohn-Sham states and the states for correction with $\mathrm{DFT}+U$, has been recently demonstrated ${ }^{19-21}$ and it is with this implementation that we perform simulations of a realistic model of the Mb heme protein (1007 atoms) in complex with two ligands, $\mathrm{CO}$ and $\mathrm{O}_{2}$, at a fully QM level.

In 1, we compare the structural properties of the three computational models shown in 1 extracted from both $\mathrm{Mb}-\mathrm{CO}$ and $\mathrm{Mb}-\mathrm{O}_{2} \mathrm{X}$-ray crystal structures ${ }^{22}$ and optimized using DFT, with a Hubbard parameter of $U=0 \mathrm{eV}$. The largest systems require the optimization of 113 atoms within a 894 atom protein environment (Computational Methods). The modeled geometries are, in general, in good agreement with each other and with the experimental structures, and our $\mathrm{Mb}-\mathrm{O}_{2}$ geometries compare favorably with optimized DFT/MM structures employing a range of different exchange-correlation functionals. ${ }^{23}$ The Fe-C-O bond angle decreases by $\sim 5^{\circ}$ on the addition of the neighboring protein residues, $\mathrm{H} 64$ and V68, while the $\mathrm{O}_{2}$ molecule retains a bent conformation of $\sim 120^{\circ}$. The root mean square deviation (RMSD) of the computed atomic positions of the heme structures from those in the experimental structures are low and most of the RMSD increase in the 53-residue system is due to the propionate side chains. Residue H64 is observed to adopt multiple conformations in crystal structures of $\mathrm{Mb}-\mathrm{O}_{2} \cdot{ }^{22,24}$ We have found the more distant conformation $\left(\mathrm{O}-\mathrm{N}_{\mathrm{H} 64}\right.$ distance of $\left.2.97 \AA\right)$ to be the more energetically favorable conformation in the 3-residue $\mathrm{Mb}-\mathrm{O}_{2}$ simulation (by $1.9 \mathrm{kcal} / \mathrm{mol}$ ) and results reported in this letter are specifically for 
this conformation. Following complete relaxation in the 53-residue system, the $\mathrm{O}-\mathrm{N}_{\mathrm{H} 64}$ distance is intermediate between the two reported experimental values. $\underline{\underline{22}}$

Table 1: Structural data for computational models, with an increasing number of protein residues, together with experimental structures. $\mathrm{X}=\mathrm{C}, \mathrm{O}$ for $\mathrm{Mb}-\mathrm{CO}$ and $\mathrm{Mb}-\mathrm{O}_{2}$ respectively. Histidine residues are labeled in 1 , The root mean square deviation of the computed atomic positions from those in the experimental structures (RMSD) is measured for non-hydrogen atoms of the heme group and ligand. Two alternative ligand to $\mathbf{N}_{\mathrm{H} 64}$ distances are quoted for the $1 \mathrm{A6M}$ crystal structure, corresponding to two equally occupied conformations of the distal histidine. Distances are measured in $\AA$.

\begin{tabular}{|c|c|c|c|c|c|c|c|c|}
\hline & \multicolumn{4}{|c|}{$\mathrm{Mb}-\mathrm{CO}$} & \multicolumn{4}{|c|}{$\mathrm{Mb}-\mathrm{O}_{2}$} \\
\hline & 1 & 3 & 53 & $1 \mathrm{~A} 6 \mathrm{G}$ & 1 & 3 & 53 & $1 \mathrm{~A} 6 \mathrm{M}$ \\
\hline $\mathrm{Fe}-\mathrm{X}$ & 1.79 & 1.78 & 1.77 & 1.82 & 1.92 & 1.87 & 1.86 & 1.81 \\
\hline$X-O$ & 1.16 & 1.16 & 1.16 & 1.09 & 1.24 & 1.26 & 1.27 & 1.24 \\
\hline $\mathrm{X}-\mathrm{N}_{\mathrm{H} 64}$ & - & 3.47 & 3.46 & 3.42 & - & 3.09 & 3.01 & $3.08 / 3.02$ \\
\hline $\mathrm{O}-\mathrm{N}_{\mathrm{H} 64}$ & - & 3.25 & 3.33 & 3.16 & - & 2.87 & 2.78 & $2.97 / 2.67$ \\
\hline $\mathrm{Fe}-\mathrm{N}_{\mathrm{H} 93}$ & 2.08 & 2.09 & 2.10 & 2.06 & 2.14 & 2.08 & 2.06 & 2.06 \\
\hline $\mathrm{Fe}-\mathrm{N}_{\text {heme }}$ & 2.03 & 2.04 & 2.03 & 1.99 & 2.03 & 2.03 & 2.03 & 2.01 \\
\hline $\mathrm{Fe}-\mathrm{X}-\mathrm{O}$ & $175.0^{\circ}$ & $170.2^{\circ}$ & $170.3^{\circ}$ & $171.1^{\circ}$ & $120.9^{\circ}$ & $120.3^{\circ}$ & $120.4^{\circ}$ & $122.5^{\circ}$ \\
\hline RMSD & 0.07 & 0.07 & 0.11 & - & 0.07 & 0.06 & 0.10 & - \\
\hline
\end{tabular}

The strength of the hydrogen bond between the distal histidine H64 and the two ligands is expected to depend strongly on the charge transfer from the heme group to the ligand. 2 reveals that the magnitude of the natural bond orbital charge population ${ }^{25}$ on $\mathrm{CO}$ is less than $0.1 e$ in all models studied. In contrast, there is substantial charge transfer to the $\mathrm{O}_{2}$ ligand, which increases with system size and reaches $-0.46 e$ in the largest system, in agreement with previous CASSCF/MM calculations. $\underline{23}$

If the dominant ligand discrimination mechanism in myoglobin were due to ligand-H64 hydrogenbonding, then we would expect the larger model proteins, in which the charge transfer to $\mathrm{O}_{2}$ is greater, to discriminate more strongly in favor of $\mathrm{O}_{2}$. Indeed, in the absence of the DFT $+U$ correction, the relative affinity of the heme group for $\mathrm{CO}$ is reduced in the 3-residue model, and reduced further in the 53-residue model (2A). The protein effect, defined as the difference between the relative binding energies of $\mathrm{CO}$ and $\mathrm{O}_{2}$ to the heme group calculated in vacuum and within the 
Table 2: Charges on the Fe ion and ligand calculated using natural population analysis, expressed in units of the electronic charge, calculated at $U=0 \mathrm{eV}$. Results vary by less than $0.06 e$ with the application of the Hubbard $U$ (up to $5 \mathrm{eV}$ ) and the Mulliken charges show the same dependency on ligand type and system size (Table S1).

\begin{tabular}{|c|c|c|c|c|}
\hline & \multicolumn{2}{|c|}{$\mathrm{Mb}-\mathrm{CO}$} & \multicolumn{2}{|c|}{$\mathrm{Mb}-\mathrm{O}_{2}$} \\
\hline & $\mathrm{Fe}$ & $\mathrm{CO}$ & $\mathrm{Fe}$ & $\mathrm{O}_{2}$ \\
\hline 1 & 0.95 & -0.05 & 1.21 & -0.30 \\
\hline 3 & 0.96 & -0.09 & 1.13 & -0.37 \\
\hline 53 & 0.39 & 0.01 & 0.82 & -0.46 \\
\hline
\end{tabular}

protein model system, is $3.7 \mathrm{kcal} / \mathrm{mol}$ and $2.4 \mathrm{kcal} / \mathrm{mol}$ in the 53 -residue and 3 -residue systems respectively. Although there is no equivalent experimental probe of the effect of the protein on the relative enthalpies of binding that we have measured here, the effect of the protein on the relative free energy of binding is inferred to be $\sim 4 \mathrm{kcal} / \mathrm{mol}$ from equilibrium constants. $\frac{1}{*}$ A rigorous comparison between the two results would require estimates of the effect of the protein on the relative entropy of binding of the two molecules, which is usually assumed to be small, and solvation effects, though the heme binding site would seem to be well-isolated from any electrostatic interaction with the solvent. A promising avenue for further work is the investigation of the effects of finite temperature sampling of protein side chain conformations on the relative binding energies. ${ }^{26,27}$ However, encouraged by the similarity between our results and the experimental protein effect subject to the caveats discussed, we make the assumption that our static ground state calculations are indicative of the finite temperature discrimination mechanism..$^{4}$

In order to examine the discrimination mechanism, we have decomposed the protein effect into distal interaction and strain energies that involve the protein fragment containing the residues H64 and V68 (Supporting Information). The first effect is the distal relative interaction energy between the heme-ligand complex and the protein fragment containing H64 and V68 and reflects the overall trend in discrimination - these interactions favor $\mathrm{O}_{2}$ binding by $3.6 \mathrm{kcal} / \mathrm{mol}$ in the 53 -residue system and by just $1.7 \mathrm{kcal} / \mathrm{mol}$ in the minimal 3-residue system. These results are consistent with experimental ${ }^{7}$ and DFT-simulated ${ }^{4}$ site-directed mutagenesis of H64, which imply that the 
hydrogen bond contributes 3.7 or $3.3 \mathrm{kcal} / \mathrm{mol}$, respectively, to ligand discrimination, and with QM/MM simulations,,$\frac{5}{}$ which give the difference in hydrogen bond strengths to be $5 \mathrm{kcal} / \mathrm{mol}$. The second effect - the distal relative strain energy stored in the protein fragment (the energy difference between the optimized $\mathrm{Mb}-\mathrm{CO}$ and $\mathrm{Mb}-\mathrm{O}_{2}$ structures with the heme group, ligand and the remainder of the protein removed) - is more difficult to estimate using conventional, cubicscaling DFT or QM/MM calculations, requiring a method sensitive to atomic displacements over a large region. In the 3-residue model, H64 and V68 store $0.3 \mathrm{kcal} / \mathrm{mol}$ of strain energy when binding $\mathrm{CO}$ (relative to $\mathrm{O}_{2}$ ). By fully optimizing the heme group and surrounding residues within the constraints of the full protein environment with first principles QM, we find that the distal relative strain falls to $-0.1 \mathrm{kcal} / \mathrm{mol}$ in the 53 -residue system, implying that the strain is not only dissipated by allowing rearrangement of the surrounding matrix, but also that there is a slight steric preference for $\mathrm{CO}$ binding. This implies that formation of the $\mathrm{H} 64-\mathrm{O}_{2}$ hydrogen bond may incur a strain penalty in the protein matrix, an effect which is not measurable using the minimal model. In both cases, however, the energy stored is much smaller than the polar discrimination effect and, as is generally assumed, $\stackrel{2}{=}$ is of the same order as the CO distortion energy. It should be emphasized that, within the 53-residue model, only the heme group, ligand and three residues around the binding site have been re-optimized upon $\mathrm{CO}$ binding, thus concentrating the strain energy difference to this local region. However, extending the relaxations to allow the optimization of eight residues (H64V68 and S92-A94) gave no change in the distal relative interaction energy $(3.6 \mathrm{kcal} / \mathrm{mol})$ and a distal relative strain of $-0.3 \mathrm{kcal} / \mathrm{mol}$, implying that both quantities are converged. The likelihood is that this optimization region would need to be extended in less rigid protein structures, though the details of the convergence of the elastic energy with system size is beyond the scope of this work.

Despite the agreement between the nature of the protein effect calculated here and the literature consensus, little is known about how the protein responds to the treatment of electron localization at the metal center. For example, the tendency of local exchange-correlation functionals to over-delocalize electrons in correlated systems leads to over-estimation of metal-ligand bind- 
A

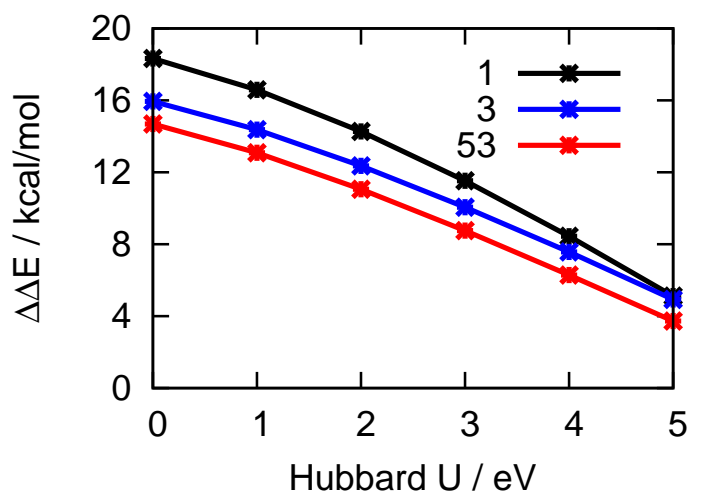

$\mathrm{B}$

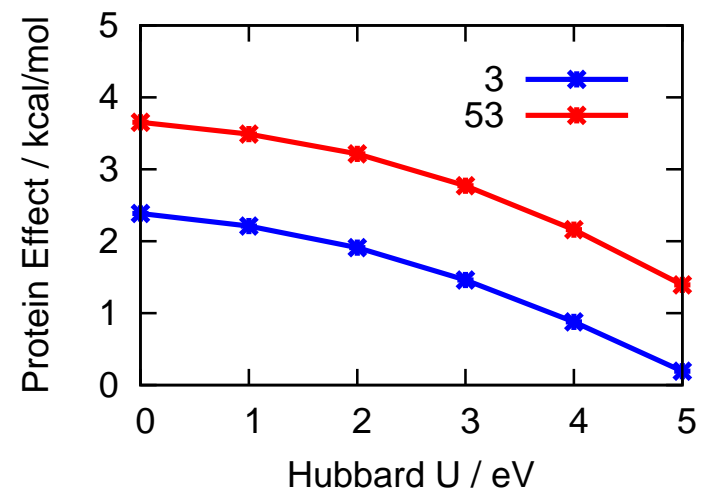

Figure 2: (A) Relative binding energies of $\mathrm{O}_{2}$ and $\mathrm{CO}$ to heme models containing increasing numbers of protein residues (positive values indicate that $\mathrm{CO}$ binding is favored). The protein decreases the preference for $\mathrm{CO}$ binding, as expected. (B) The magnitude of this protein effect on ligand discrimination as a function of the Hubbard $U$ parameter. Dashed lines are estimated from experimental equilibrium constants. $\frac{1}{}$

ing energies. In agreement with previous $\mathrm{DFT}+U$ calculations, $\underline{\underline{28}}$ we find that the addition of the localized self-interaction correction recovers the quintuplet electronic ground state of the isolated heme group and improves the local magnetic moments and, to some extent, Fe-ligand binding energies (Figures S1 and S2). With this improved description of the Fe $3 d$ orbital occupancies, the relative affinity of heme for $\mathrm{CO}$ is reduced, both with and without the protein environment (2A), and the protein effect, correspondingly, decreases somewhat with increasing $U(2 \mathrm{~B})$. However, this variation is all in the treatment of the heme/ligand sub-system, as expected from an intrinsically short-ranged correction. The distal relative interaction energy varies much less (between 3.6 and $4.3 \mathrm{kcal} / \mathrm{mol}$, Figure S3) and the distal relative strain is, by definition, unchanged.

We have demonstrated that linear-scaling DFT may be applied to a heme group embedded in a realistic model of the heme protein, consisting of over 1000 atoms, and used to elucidate features of a much-discussed discrimination mechanism. Compared to the minimal model of the heme-protein interaction, the 53-residue model of $\mathrm{Mb}-\mathrm{O}_{2}$ has a larger charge transfer to the ligand, which results in a stronger hydrogen-bonding interaction with the distal side of the protein. In comparison, the relative strain energy stored in the protein is smaller, and is certainly not enough to account for the ligand discrimination effect in myoglobin. While a systematic study of the convergence 
of energetic properties of heme with system size, the finite temperature sampling of protein side chain conformations, and a complete description of correlated electron effects in ligand-heme interactions, remain as future avenues of investigation, our work represents an encouraging, positive result for the feasibility of the use of large-scale DFT simulations in a well-studied benchmark system. The treatment of entire proteins at the full QM level is now becoming widespread, $11,12,29$ potentially increasing the accuracy and range of problems that are open to study in fields ranging from small molecule therapeutics to enzymology.

\section{Computational Method}

QM calculations were performed with spin-polarized DFT as implemented in the ONETEP code, $\underline{\underline{8}}$ using the PBE gradient corrected exchange-correlation functional. $\underline{30}$ Van der Waals interactions were approximated by augmenting the DFT energy functional with damped London potentials with parameters optimized specifically for the PBE functional. $\underline{9}$ The ONETEP parameters used are described in the Supporting Methods. Computational models of the heme site were based on Xray crystal structures of sperm whale $\mathrm{Mb}$ in two different ligation states: oxygenated $\left(\mathrm{Mb}-\mathrm{O}_{2}\right.$, PDB: 1A6M) and carbonmonoxygenated (Mb-CO, PDB: 1A6G). ${ }^{22}$ Three different sized systems were constructed by retaining one (H93), three (H64, V68 and H93) or 53 (I28-F46, K63-L72, P88-I111) residues surrounding the heme group in the two X-ray crystal structures. Geometry optimization $^{31}$ was performed to tolerances of $0.003 \mathrm{eV}$ and $0.05 \mathrm{eV} / \AA$ on the total energies and forces respectively. Initial geometry optimization of the 53-residue $\mathrm{Mb}-\mathrm{O}_{2}$ structure revealed substantial spin populations on four charged residues on the exterior of the model system (K34, E41, D44 and K63). These residues would either form salt-bridges or would be solvent-exposed in their real environment, but are exposed to the vacuum in our model system. We therefore manually transferred protons from K34 and K63 to E41 and D44 in order to neutralize these residues and reduce the spurious local magnetic moments to zero. Following further optimization to a force tolerance of $0.2 \mathrm{eV} / \AA$, the outer 50 residues were frozen and the heme group and three closest 
residues (H64, V68 and H93) were optimized to the tolerances described above. Mb-CO was modeled by replacing the heme group and residues H64, V68 and H93 by their positions in the carbonmonoxygenated crystal structure, and allowing them to relax in the identical frozen 50residue environment. This scheme ensures that energy differences are directly attributable to local changes in the binding site, whilst accounting for long-ranged polarization and constraints of the protein scaffold. It is important, however, to ensure that results are converged with respect to the size of the relaxed region.

To correct for the underestimation of $3 d$ electron localization by the approximate exchangecorrelation functional, a rotationally-invariant form of the widely-used DFT $+U$ correction 14,15 was applied to the Fe $3 d$ manifold. In this method, implemented in the linear-scaling DFT formalism,,$\frac{19-21}{2}$ and previously applied to heme systems with success, $, 28,32$ an additional term is added to the energy functional, taking the form for a single Fe ion:

$$
E_{U}=\frac{U}{2} \sum_{\sigma} \sum_{m}\left[n_{m m}^{\sigma}-\sum_{m^{\prime}} n_{m m^{\prime}}^{\sigma} n_{m^{\prime} m}^{\sigma}\right], \quad \text { where } \quad n_{m m^{\prime}}^{\sigma}=\sum_{i}\left\langle\varphi_{m} \mid \psi_{i}^{\sigma}\right\rangle f_{i}^{\sigma}\left\langle\psi_{i}^{\sigma} \mid \varphi_{m^{\prime}}\right\rangle
$$

Here, the functions $\left|\psi_{i}^{\sigma}\right\rangle$ are Kohn-Sham orbitals of spin $\sigma$ and occupancy $f_{i}^{\sigma}$. The $\left|\varphi_{m}\right\rangle$ are the Hubbard projectors that delineate the subspace for correction, in this case, iron-centered atomic $3 d$ orbitals that are numerically solved using the corresponding atomic pseudopotential. The Hubbard $U$ determines the strength of the $\mathrm{DFT}+U$ correction, and while it may be computed from first principles using a number of different methods, it is treated as a free parameter in our calculations. The net effect, with increasing $U$, is to more strongly penalize deviation from integer values of the eigenvalues of the $3 d$ subspace occupancy matrices $n_{m m^{\prime}}^{\sigma}$ and correct for localized self-interaction errors in the approximate functional. 14,15 The DFT $+U$ ground state wave-function remains single-determinantal, with no finite temperature or explicitly dynamical quantum effects included, although the extension of $\mathrm{DFT}+U$ to dynamical mean-field theory, offering improved descriptions of excited-state quantities such as optical spectra, has recently been applied to heme (unpublished results). In order to describe the dominant open-shell singlet ground state of $\mathrm{Mb}-\mathrm{O}_{2}$ 
within Kohn-Sham DFT, it was necessary to break the magnetic symmetry by applying effective magnetic fields, of opposite sign, locally to the $\mathrm{Fe} 3 d$ and $\mathrm{O}_{2} 2 p$ manifolds. These effective fields were implemented by means of an extension of the $\mathrm{DFT}+U$ functionality, and were switched off automatically when the electronic density began to converge. The independence of the total energy with respect to the strength of the applied fields was carefully tested. All calculations at $\mathrm{DFT}+U>0 \mathrm{eV}$ were performed on converged $\mathrm{DFT}+U=0 \mathrm{eV}$ structures, which introduced an estimated error of less than $0.2 \mathrm{kcal} / \mathrm{mol}$ (Table S2).

\section{Acknowledgement}

We are grateful to Nicholas Hine for helpful discussions. Computational resources were provided by the Cambridge HPC Service, funded by EPSRC Grant EP/F032773/1. DJC, DDO'R and MCP acknowledge support from the EPSRC.

\section{Supporting Information Available}

Supporting methods, calculation of distal interaction and strain energies, Mulliken charge analysis, energetics of $\mathrm{FeP}(\mathrm{Im})$, variation of local magnetic moments and distal interaction energy with $U$, and tests of our geometry optimization protocol at non-zero Hubbard $U$. This material is available free of charge via the Internet at http://pubs.acs.org//

\section{References}

(1) Olson, J. S.; Phillips Jr., G. N. Myoglobin Discriminates Between $\mathrm{O}_{2}$, NO, and CO by Electrostatic Interactions with the Bound Ligand. J. Biol. Inorg. Chem. 1997, 2, 544-522.

(2) Spiro, T. G.; Kozlowski, P. M. Is the CO Adduct of Myoglobin Bent, and Does It Matter? Acc. Chem. Res. 2001, 34, 137-144.

(3) Collman, J. P.; Brauman, J. I.; Halbert, T. R.; Suslick, K. S. Nature of $\mathrm{O}_{2}$ and CO Binding to Metalloporphyrins and Heme Proteins. Proc. Natl. Acad. Sci. U.S.A. 1976, 73, 3333-3337. 
(4) De Angelis, F.; Jarzȩcki, A. A.; Car, R.; Spiro, T. G. Quantum Chemical Evaluation of Protein Control over Heme Ligation: $\mathrm{CO} / \mathrm{O}_{2}$ Discrimination in Myoglobin. J. Phys. Chem. B 2005, $109,3065-3070$.

(5) Sigfridsson, E; Ryde, U. Theoretical Study of the Discrimination between $\mathrm{O}_{2}$ and CO by Myoglobin. J. Inorg. Biochem 2002, 91, 101-115.

(6) Phillips Jr., G. N.; Teodoro, M. L.; Li, T.; Smith, B.; Olson, J. S. Bound CO is a Molecular Probe of Electrostatic Potential in the Distal Pocket of Myoglobin. J. Phys. Chem. B 1999, $103,8817-8829$.

(7) Springer, B. A.; Sligar, S. G.; Olson, J. S.; Phillips Jr., G. N. Mechanisms of Ligand Recognition in Myoglobin. Chem. Rev. 1994, 94, 699-714.

(8) Skylaris, C. K.; Haynes, P. D.; Mostofi, A. A.; Payne, M. C. Introducing OnETEP: LinearScaling Density Functional Simulations on Parallel Computers. J. Chem. Phys. 2005, 122, 084119.

(9) Hill, Q.; Skylaris, C. K. Including Dispersion Interactions in the ONETEP Program for Linear-Scaling Density Functional Theory Calculations. Proc. R. Soc. A 2009, 465, 669-683.

(10) Hine, N. D. M.; Haynes, P. D.; Mostofi, A. A.; Skylaris, C. K.; Payne, M. C. Linear-Scaling Density-Functional Theory with Tens of Thousands of Atoms: Expanding the Scope and Scale of Calculations with ONETEP. Comp. Phys. Comm. 2009, 180, 1041-1053.

(11) Cole, D. J.; Skylaris, C. K.; Rajendra, E.; Venkitaraman, A. R.; Payne, M. C. Protein-Protein Interactions from Linear-Scaling First-Principles Quantum-Mechanical Calculations. EPL 2010, 91, 37004.

(12) Cole, D. J.; Rajendra, E.; Roberts-Thomson, M.; Hardwick, B.; McKenzie, G. J.; Payne, M. C.; Venkitaraman, A. R.; Skylaris, C. K. Interrogation of the Protein-Protein Inter- 
actions between Human BRCA2 BRC Repeats and RAD51 Reveals Atomistic Determinants of Affinity. PLoS Comp. Bio. 2011, 7, e1002096.

(13) Cohen, A. J.; Mori-Sánchez, P.; Yang, W. Insights into Current Limitations of Density Functional Theory. Science 2008, 321, 792-794.

(14) Cococcioni, M.; de Gironcoli, S. Linear Response Approach to the Calculation of the Effective Interaction Parameters in the LDA $+U$ Method. Phys. Rev. B 2005, 71, 035105.

(15) Pickett, W. E.; Erwin, S. C.; Ethridge, E. C. Reformulation of the LDA $+U$ Method for a Local-Orbital Basis. Phys. Rev. B 1998, 58, 1201-1209.

(16) Kulik, H. J.; Cococcioni, M.; Scherlis, D. A.; Marzari, N. Density-Functional Theory in Transition-Metal Chemistry: A Self-Consistent Hubbard U Approach. Phys. Rev. Lett. 2006, 97, 103001.

(17) Kulik, H. J.; Marzari, N. A Self-Consistent Hubbard $U$ Density-Functional Theory Approach

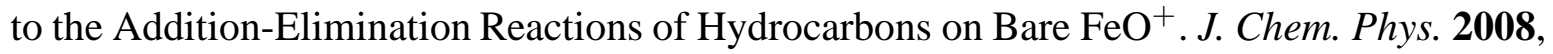
$129,134314$.

(18) Kulik, H. J.; Marzari, N. Transition-Metal Dioxides: A Case for the Intersite Term in Hubbard-Model Functionals. J. Chem. Phys. 2011, 134, 094103.

(19) O’Regan, D. D.; Hine, N. D. M.; Payne, M. C.; Mostofi, A. A. Projector Self-Consistent DFT $+U$ using Non-Orthogonal Generalized Wannier Functions. Phys. Rev. B 2010, 82, 081102(R).

(20) O’Regan, D. D.; Payne, M. C.; Mostofi, A. A. Subspace Representations in ab initio Methods for Strongly Correlated Systems. Phys. Rev. B 2011, 83, 245124.

(21) O’Regan, D. D.; Hine, N. D. M.; Payne, M. C.; Mostofi, A. A. Linear-Scaling DFT+U with Full Local Orbital Optimization. Phys. Rev. B 2012, 85, 085107. 
(22) Vojtěchovský, J.; Chu, K.; Berendzen, J.; Sweet, R. M.; Schlichting, I. Crystal Structures of Myoglobin-Ligand Complexes at Near-Atomic Resolution. Biophys. J. 1999, 77, 2153-2174.

(23) Chen, H.; Ikeda-Saito, M.; Shaik, S. Nature of the Fe-O ${ }_{2}$ Bonding in Oxy-Myoglobin: Effect of the Protein. J. Am. Chem. Soc. 2008, 130, 14778-14790.

(24) Unno, M.; Chen, H.; Kusama, S.; Shaik, S.; Ikeda-Saito, M. Structural Characterization of the Fleeting Ferric Peroxo Species in Myoglobin: Experiment and Theory. J. Am. Chem. Soc. 2008, 129, 13394-13395.

(25) Reed, A. E.; Weinstock, R. B.; Weinhold, F. Natural Population Analysis. J. Chem. Phys. $1985,83,735-746$.

(26) Alcantara, R. E.; Xu, C.; Spiro, T. G.; Guallar, V. A Quantum Chemical Picture of Hemoglobin Affinity. Proc. Natl. Acad. Sci. USA 2007, 104, 18451-18455.

(27) Strickland, N.; Mulholland, A. J.; Harvey, J. N. The Fe-CO Bond Energy in Myoglobin: A QM/MM Study of the Effect of Tertiary Structure. Biophys. J. 2006, 90, L27-L29.

(28) Scherlis, D. A.; Cococcioni, M.; Sit, P.; Marzari, N. Simulation of Heme using DFT+U: A Step toward Accurate Spin-State Energetics. J. Phys. Chem. B 2007, 111, 7384-7391.

(29) Ufimtsev, I. S.; Luehr, N.; Martinez, T. J. Charge Transfer and Polarization in Solvated Proteins from ab initio Molecular Dynamics. J. Phys. Chem. Lett. 2011, 2, 1789-1793.

(30) Perdew, J. P.; Burke, K.; Ernzerhof, M. Generalized Gradient Approximation Made Simple. Phys. Rev. Lett. 1996, 77, 3865-3868.

(31) Hine, N. D. M.; Robinson, M.; Haynes, P. D.; Skylaris, C. K.; Payne, M. C.; Mostofi, A. A. Accurate Ionic Forces and Geometry Optimisation in Linear Scaling Density-Functional Theory with Local Orbitals. Phys. Rev. B 2011, 83, 195102.

(32) Sena, A. M. P.; Brázdová, V.; Bowler, D. R. Density Functional Theory Study of the IronBased Porphyrin Haem(b) on the Si(111):H Surface. Phys. Rev. B 2009, 79, 245404. 


\title{
Supporting Information for:
}

\section{Ligand Discrimination in Myoglobin from Linear-Scaling DFT+ $U$}

\author{
Daniel J. Cole, ${ }^{\dagger}$ David D. O’Regan ${ }^{\ddagger}$ and Mike C. Payne ${ }^{*, \dagger, \text { II }}$ \\ Theory of Condensed Matter Group, Cavendish Laboratory, University of Cambridge, J. J. \\ Thomson Avenue, Cambridge CB3 OHE, UK., Theory and Simulation of Materials, École \\ Polytechnique Fédérale de Lausanne, MXC 341, Station 12, CH-1015 Lausanne, Switzerland., \\ and \\ E-mail: mcp1@cam.ac.uk
}

\footnotetext{
${ }^{*}$ To whom correspondence should be addressed

†University of Cambridge

†École Polytechnique Fédérale de Lausanne

${ }^{\mathbb{I}}$ This document is the Accepted Manuscript version of a Published Work that appeared in final form in J. Phys. Chem. Lett., 2012, 3 (11), pp 1448-1452, copyright (C) American Chemical Society after peer review and technical editing by the publisher.

To access the final edited and published work see http://pubs.acs.org/doi/full/10.1021/jz3004188
} 


\section{Supporting Methods}

\section{Computational Models}

Computational models of the heme site were extracted from X-ray crystal structures of sperm whale $\mathrm{Mb}$ in oxygenated (Mb- $\mathrm{O}_{2}$, PDB: 1A6M) and carbonmonoxygenated (Mb-CO, PDB: 1A6G) ligation states. Waters of crystallization and sulfate ions were removed. Oxygenated and carbonmonoxygenated 1- and 3-residue systems were extracted from the relevant crystal structure and modelled with the propionate side chains hydrogen-terminated, giving zero net charge. Histidine and valine residues were terminated at the $\mathrm{C}_{\gamma}$ and $\mathrm{C}_{\beta}$ atoms, respectively, and these atoms were held fixed at their crystal positions in the 3-residue simulation in order to model the constraints of the protein environment. The 53-residue system was extracted from the $\mathrm{Mb}-\mathrm{O}_{2}$ crystal structure, with protein chains terminated by acetyl and methylamino groups (using the AMBER10 package $\underline{1}$ ) and with all amino acids except histidine in their normal protonation states at $\mathrm{pH}$ 7. The assignment of histidine protonation states were taken from the literature $\underline{\underline{2}}$ and, in particular, H64 was modelled as the $\varepsilon$-tautomer. The resulting complex consisted of a total of 1007 atoms, with a net charge of $+2 e$.

\section{ONETEP Parameters}

The ONETEP program achieves computational cost that scales linearly with the number of atoms by exploiting the "near-sightedness" of the single-particle density matrix in non-metallic systems, $\underline{3}$ though no density matrix truncation was applied in our calculations in order to maximize accuracy. The density matrix is expressed in terms of a set of nonorthogonal generalized Wannier functions $(\mathrm{NGWFs})^{4}$ that are localized in real space, with truncation radii set to $4.8 \AA$, and optimized during the process of total energy minimization. The NGWFs were initialized to numerical orbitals obtained from solving the Kohn-Sham equation for free atoms, with a $1 s$ configuration for $\mathrm{H}$, a $2 s 2 p$ configuration for $\mathrm{C}, \mathrm{N}$ and $\mathrm{O}$, and a $3 d 4 s 4 p$ configuration for Fe. $\frac{\sqrt{5}}{\text { The NGWFs }}$ were expanded in a basis of periodic cardinal sine (psinc) functions $\underline{\underline{6}}$ with a kinetic energy cut-off 
of $920 \mathrm{eV}$. The protein effect was estimated to be converged to less than $0.5 \mathrm{kcal} / \mathrm{mol}$ with respect to changes in NGWF radii and psinc energy cut-off. Core electrons were described using norm-conserving pseudopotentials, including a non-linear core correction for that of the Fe ion. The spherical cut-off approach for Coulomb potentials was used to eliminate all interactions of the molecules with their periodic images. $\underline{\underline{7}}$ Dispersion interactions were included by augmenting the DFT energy expression by damped London potentials with parameters optimized specifically for the PBE functional. $\underline{8}$ The root mean square error in gas phase binding energies of a benchmark set of complexes calculated using the DFT methodology described above has been shown to be approximately $1 \mathrm{kcal} / \mathrm{mol}$ when compared to MP2 and $\operatorname{CCSD}(\mathrm{T})$ methods extrapolated to the complete basis set limit. $\underline{8}$ Atomic charges and spin populations were calculated using Mulliken and natural bond orbital analyses implemented within ONETEP. 9,10

\section{Distal Interaction Energy and Strain}

The protein effect on the relative binding energies of $\mathrm{CO}$ and $\mathrm{O}_{2}$ to the heme group consists of: direct interactions between the heme-ligand subsystem and the protein, distortions of the protein by the heme-ligand subsystem and distortions of the heme-ligand subsystem by the protein. The protein effect in Figure 2B contains all of these effects. The last contribution, describing changes in transition metal-ligand geometries, is the least accurate (even with the Hubbard $U$ correction), but also the most studied using minimal models and more accurate descriptions of correlated electron effects. $\frac{11,12}{}$ For these reasons, we have decomposed the protein effect to describe the first two contributions. Namely, the distal relative interaction energy is:

$$
\Delta \Delta E=\Delta E(\text { total })-\Delta E(\mathrm{~K} 63-\mathrm{L} 72)-\Delta E(\text { heme, ligand, } \mathrm{I} 28-\mathrm{F} 46, \mathrm{P} 88-\mathrm{I} 111) .
$$

$\Delta E$ (total) is the difference in total energy between the optimized $\mathrm{CO}$ and $\mathrm{O}_{2}$ complexes. K63-L72 is the protein fragment containing distal residues H64 and V68 and the remainder of the complex is described by the last term in the equation. We choose the distal interaction energy so that it may 
be compared with experimental H64 mutagenesis studies and to remove inaccuracies in the description of the Fe-H93 interaction. Finally, the distal relative strain is defined as $\Delta E(\mathrm{~K} 63-\mathrm{L} 72)$.

Table S1. Charges on the Fe ion and ligand from Mulliken analysis in the NGWF basis in units of the electronic charge.

\begin{tabular}{|c|c|c|c|c|}
\hline & \multicolumn{2}{|c|}{$\mathrm{Mb}-\mathrm{CO}$} & \multicolumn{2}{|c|}{$\mathrm{Mb}-\mathrm{O}_{2}$} \\
\hline & $\mathrm{Fe}$ & $\mathrm{CO}$ & $\mathrm{Fe}$ & $\mathrm{O}_{2}$ \\
\hline 1 & 1.20 & -0.12 & 1.27 & -0.26 \\
\hline 3 & 1.17 & -0.16 & 1.19 & -0.41 \\
\hline 53 & 0.22 & 0.06 & 0.79 & -0.58 \\
\hline
\end{tabular}
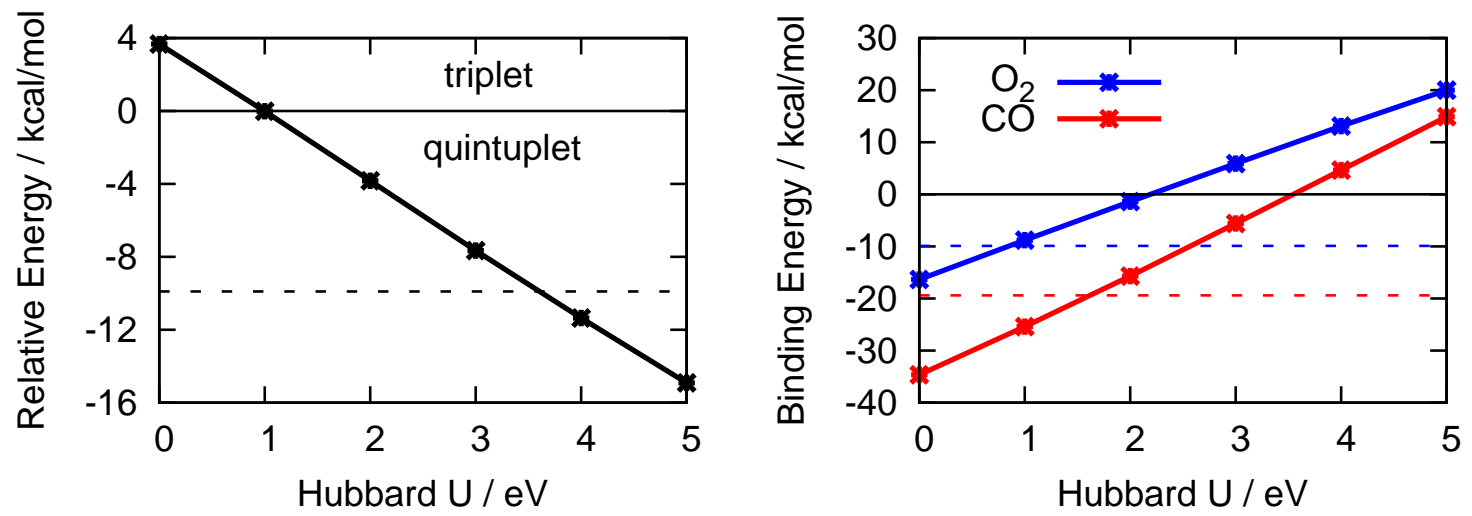

Figure S1. (left) Relative energies of triplet and quintuplet spin states of FeP(Im) extracted from the unligated X-ray crystal structure (PDB: $1 \mathrm{~A} 6 \mathrm{~N}^{13}$ ). The experimental quintuplet ground state is favored at $U>1 \mathrm{eV}$. (right) Binding energies of $\mathrm{O}_{2}$ and $\mathrm{CO}$ ligands to $\mathrm{FeP}(\mathrm{Im})$ in vacuum. Binding is over-estimated in the absence of the $\mathrm{DFT}+U$ correction. Dashed lines show the respective CASPT2 results. $\underline{14}$ 

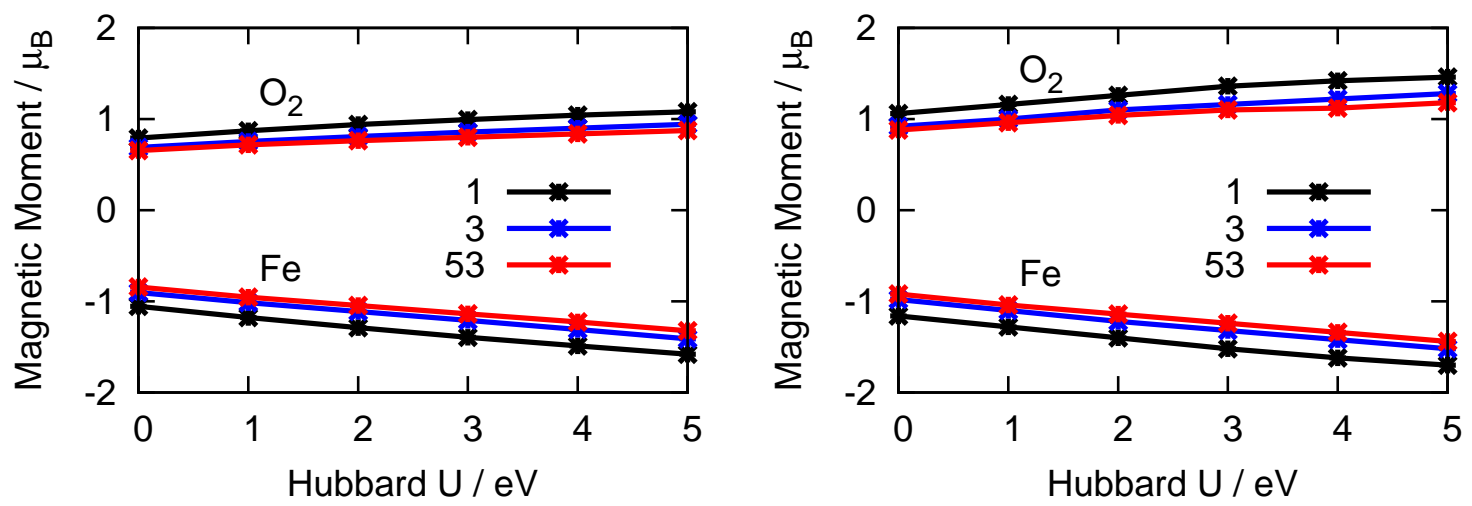

Figure S2. Local magnetic moments on the iron and $\mathrm{O}_{2}$ species in models of increasing numbers of residues, consistent with an open-shell singlet spin state. The moments are calculated using (left) the DFT $+U$ occupancy matrices (Equation 1) and (right) Mulliken analysis in the NGWF basis.

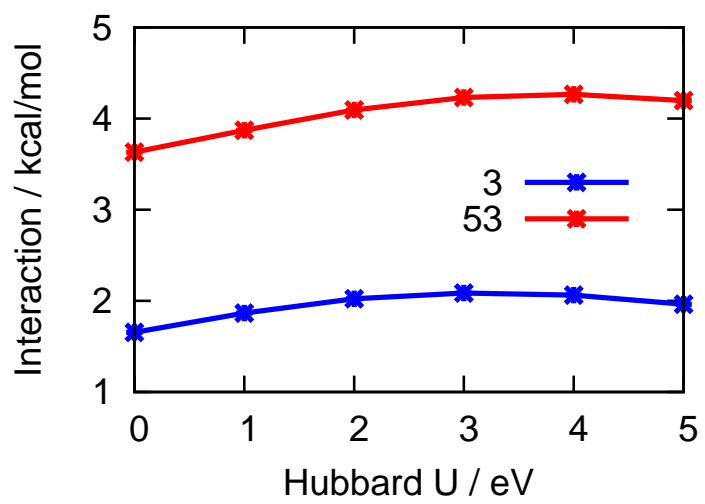

Figure S3. Variation of distal relative interaction energy with the DFT $+U$ parameter. The former is mostly long-ranged, while the latter determines the strength of an intrinsically short-ranged selfinteraction correction. 
Table S2. Relative binding energies of $\mathrm{CO}$ and $\mathrm{O}_{2}$ to two heme models with increasing numbers of protein residues $(\Delta \Delta \mathbf{E})$, at $U=3.0 \mathrm{eV}$, for two different protocols. Geometry optimize indicates that the structures have been re-optimized to a force tolerance of $0.15 \mathrm{eV} / \AA$, at $U=3.0 \mathrm{eV}$, and single point indicates that a single calculation with $U=3.0 \mathrm{eV}$ has been performed on the structure optimized at $U=0 \mathrm{eV}$. The maximum error introduced by not re-optimizing is less than $0.2 \mathrm{kcal} / \mathrm{mol}$.

\begin{tabular}{lccc} 
& \multicolumn{2}{c}{$\Delta \Delta \mathrm{E} / \mathrm{kcal} / \mathrm{mol}$} & \\
\cline { 2 - 3 } & & & \\
& 1 residue & 3 residues & Protein Effect \\
\hline Geometry Optimize & 11.47 & 9.88 & 1.59 \\
Single Point & 11.52 & 10.06 & 1.46 \\
\hline
\end{tabular}




\section{References}

(1) Case, D. A. et al. 2008, Amber10, University of California, San Francisco.

(2) Strickland, N.; Mulholland, A. J.; Harvey, J. N. The Fe-CO Bond Energy in Myoglobin: A QM/MM Study of the Effect of Tertiary Structure. Biophys. J. 2006, 90, L27-L29.

(3) Prodan, E.; Kohn, W. Nearsightedness of Electronic Matter. Proc. Natl. Acad. Sci. USA 2005, $102,11635-11638$.

(4) Skylaris, C. K.; Mostofi, A. A.; Haynes, P. D.; Dieguez, O.; Payne, M. C. The NonOrthogonal Generalised Wannier Function Pseudopotential Plane-Wave Method. Phys. Rev. B 2002, 66, 035119.

(5) Ruiz-Serrano, A.; Hine, N. D. M.; Skylaris, C. K. Pulay Forces from Localized Orbitals Optimized in situ using a psinc Basis Set Submitted (2012).

(6) Mostofi, A. A.; Haynes, P. D.; Skylaris, C. K.; Payne, M. C. Preconditioned Iterative Minimisation for Linear-Scaling Electronic Structure Calculations. J. Chem Phys. 2003, 119, 8842-8848.

(7) Hine, N. D. M.; Dziedzic, J.; Haynes, P. D.; Skylaris, C. K. Electrostatic Interactions in Finite Systems Treated with Periodic Boundary Conditions: Application to Linear-Scaling Density Functional Theory. J. Chem. Phys. 2011, 135, 204103.

(8) Hill, Q.; Skylaris, C. K. Including Dispersion Interactions in the ONETEP Program for Linear-Scaling Density Functional Theory Calculations. Proc. R. Soc. A 2009, 465, 669-683.

(9) Reed, A. E.; Weinstock, R. B.; Weinhold, F. Natural Population Analysis. J. Chem. Phys. 1985, 83, 735-746.

(10) Lee, L. P.; Cole, D. J.; Payne, M. C.; Skylaris, C. K. Natural Bond Orbital Analysis in LinearScaling Density Functional Theory Calculations In preparation (2012). 
(11) Chen, H.; Ikeda-Saito, M.; Shaik, S. Nature of the Fe-O 2 Bonding in Oxy-Myoglobin: Effect of the Protein. J. Am. Chem. Soc. 2008, 130, 14778-14790.

(12) Weber, C.; O’Regan, D. D.; Hine, N. D. M.; Littlewood, P. B.; Kotliar, G.; Payne, M. C. Binding of Iron Porphyrin Driven by Valence Fluctuations In preparation (2012).

(13) Vojtěchovský, J.; Chu, K.; Berendzen, J.; Sweet, R. M.; Schlichting, I. Crystal Structures of Myoglobin-Ligand Complexes at Near-Atomic Resolution. Biophys. J. 1999, 77, 2153-2174.

(14) Radón, M.; Pierloot, K. Binding of $\mathrm{CO}$, NO, and $\mathrm{O}_{2}$ to Heme by Density Functional and Multireference ab initio Calculations. J. Phys. Chem. A 2008, 112, 11824-11832. 